\title{
Seizures in children with fever: Generally good outcome
}

Janet Jankowiak, MD; and Beth Malow, MD, MS

\begin{abstract}
Young children with high fevers may occasionally have seizures (called febrile seizures). Febrile seizures are the most common convulsive disorder of early childhood and occur in $2 \%$ to $5 \%$ of children in the United States. These seizures occur in previously healthy children from 3 months to 5 years old during an illness with a high fever, often an ear infection. Whether these children later develop epilepsy, a chronic condition with recurring seizures, is a question parents naturally ask. More information about seizures and epilepsy can be found on the following page.
\end{abstract}

Because some patients with difficult-to-control epilepsy have had febrile seizures, some scientists have questioned whether febrile seizures cause epilepsy. In particular, it is speculated that children with febrile seizures may be at increased risk of developing a focal abnormality in the brain called mesial temporal sclerosis (MTS). MTS is the most common lesion found in patients with a type of epilepsy that comes from an area of the brain called the temporal lobe. This term means "hardening" or scarring of an area of the temporal lobe close to the middle of the brain. In MTS, this region is also atrophied or shrunken, sometimes on one side more than the other. The presence of this abnormality may be responsible for recurring seizures. In this issue of Neurology, Tarkka et al. (Tarkka R, Paakko E, Pyhtinen J, Uhari M, Rantala $\mathrm{H}$. Febrile seizures and mesial temporal sclerosis-no association in $\mathrm{a}$ long-term follow-up study.) followed a group of children for 8 to 15 years after a febrile seizure.

In this study, 329 children with febrile seizures were followed after their first febrile seizure. Eight children had an unprovoked seizure after the initial febrile seizure (i.e., a seizure occurring without a fever, trauma or another, clear cause). Another 24 children had very long febrile seizures, lasting longer than 30 minutes (most febrile seizures last less than 15 minutes). These two groups of children (32 total) were speculated to be at greatest risk for developing the focal brain abnormality, mesial temporal sclerosis. A group of another 32 children, matched by age, sex, and handedness, which had only had a simple febrile seizure (lasting less than 15 minutes), was selected as the control group. These children were chosen as controls because a simple febrile seizure is unlikely to cause permanent structural brain damage. All the children were followed for 8 to 15 years after their first febrile seizure.

Each child had an MRI scan of the brain to look for any abnormalities, especially the size and shape of a particular region of the brain within the temporal lobe. The temporal lobe is an area of the brain that is especially important for memory and language. It is also an area known to produce seizures when it is damaged, as in MTS. No MTS and no significant differences in size of the area of the temporal lobe studied on MRI were found between the patients with a pro- longed first febrile seizure or an unprovoked seizure after the first febrile seizure and the control group. School performance and neurologic exams of the children with a prolonged first febrile seizure were as good as those of the controls. Even children who had multiple febrile seizures during their first episode and those with recurrent febrile seizures did not have any temporal lobe abnormalities on MRI. Children with a prolonged first febrile seizure or unprovoked seizure recurrence should be the most likely to have a bad outcome from their seizures, but did not. A particular strength of this study is that these children were followed over 12 years, on average, after their first febrile seizure. It is unlikely that any new cases with epilepsy will arise later among these children since usually the epilepsy begins during the first 9 years after a febrile seizure.

The good news from this study is that it shows that febrile seizures do not cause either neurologic sequelae or brain damage in children who have normal development and are otherwise healthy. However, families should be aware that febrile seizures may recur with later febrile illnesses and should be treated. The reason many patients with temporal lobe epilepsy have a history of febrile seizures may be that the febrile seizure is the first sign that the child is predisposed to having seizures, and not that febrile seizures cause epilepsy. Further larger scale investigations may be needed to confirm the findings in this study. 
WHAT IS A SEIZURE? A seizure is a disruption in the normal electrical activity of the brain. Normally the brain is very active, passing electrical messages back and forth between nerve cells. When a person has a seizure, there is abnormal firing of nerve cells and the messages become jumbled in part or all of the brain.

A seizure may cause a variety of different symptoms: twitching or shaking in an arm, leg, one side of the face, or the whole body; repetitive movements or gestures; confusion; feelings of fear or other emotions; hallucinations (odd smells, tastes, sounds or seeing things that are not there); loss of consciousness; and convulsions.

\section{What Causes Seizures?}

Anyone may have a seizure in certain conditions. Common causes are: fever-in young children (see Febrile Seizures, below); head trauma (during birth or any time later); infection of the brain or nervous system, e.g., meningitis; brain tumors; very low blood sugar; stroke (a brain attack); lack of oxygen to the brain; and poisoning, e.g., alcohol and various drugs.

\section{What Are Febrile Seizures?}

Febrile seizures are generally benign and occur in children from ages 3 months to 5 years, with average age at onset of 18 to 22 months. They occur in the setting of a febrile illness, usually as the fever is rising fast. They are associated with common childhood infections such as ear infections, tonsillitis, upper airway infections, and gastrointestinal infections. Most are associated with viral illnesses.
Febrile seizures are more common in boys. They also tend to run in families. About one third of children who have a first febrile seizure will have a second one, almost always within 2 years. The earlier the onset of febrile seizures, the greater chance that they will recur. However, less than $5 \%$ of children with febrile seizures go on to develop epilepsy.

Although febrile seizures are usually benign, it is critical that the child be evaluated immediately to identify the cause of the fever and treat conditions such as meningitis or intoxication.

\section{What Is Epilepsy?}

Epilepsy is an episodic recurrence of seizures that are not due to fever, active infection, drug effects, or other triggering causes. It may be caused by a variety of conditions that injure a part or all of the brain: problems in development of the brain that occurs before birth; inherited disorders of the brain or nervous system; brain trauma; brain tumors; stroke; infections; and poisoning. About $70 \%$ have no known cause.

Each year there are 125,000 new cases of epilepsy. About 2.5 million people in the United States have some form of epilepsy.

\section{How Is Epilepsy Diagnosed?}

Medical history: the doctor will need to know as much about what happened during, immediately before, and after the seizure. How often seizures occur, whether there are any warning signs, and whether the patient remembers anything about the seizure are all important. Someone who has witnessed the seizures can provide valuable in- formation that the patient may not know.

Electroencephalography (EEG) is a simple and painless study that records the brain's electrical activity picked up by tiny wires taped to the head. Specific brain wave patterns may be noted during or between seizures in patients with epilepsy and may help with diagnosis.

Imaging studies to look at the brain may be helpful in locating tumors, scars, or other abnormalities that may be causing seizures. MRI (magnetic resonance imaging) and CT (computed tomography) scans create pictures of the inside of the brain.

\section{How Is Epilepsy Treated?}

Drugs called anticonvulsants are used most commonly to treat seizures. There are a number of different medications that can be used, alone or in combination. These drugs are effective in 60 to $80 \%$ ofcases. Close supervision by a doctor is essential to watch for side effects and obtain the best seizure control.

People with epilepsy can help control seizures by avoiding alcohol and caffeine, avoiding unusual stress, getting enough sleep, taking their medications as prescribed, and working closely with their doctor. A special diet may be helpful in controlling certain types of seizures in children but requires very close medical supervision. Surgery may be very helpful when medication fails and the area of the brain where the seizure occurs is known.

\section{For More Information}

Epilepsy Foundation of America: www.efa.org 


\section{Neurology}

\section{Seizures in children with fever: Generally good outcome \\ Janet Jankowiak and Beth Malow \\ Neurology 2003;60;E1-E2 \\ DOI 10.1212/WNL.60.2.E1}

This information is current as of January 28, 2003

\section{Updated Information \&}

Services

Permissions \& Licensing

Reprints including high resolution figures, can be found at:

http://n.neurology.org/content/60/2/E1.full

Information about reproducing this article in parts (figures,tables) or in its entirety can be found online at:

http://www.neurology.org/about/about_the_journal\#permissions

Information about ordering reprints can be found online:

http://n.neurology.org/subscribers/advertise

Neurology ${ }^{\circledR}$ is the official journal of the American Academy of Neurology. Published continuously since 1951, it is now a weekly with 48 issues per year. Copyright. All rights reserved. Print ISSN: 0028-3878. Online ISSN: 1526-632X.

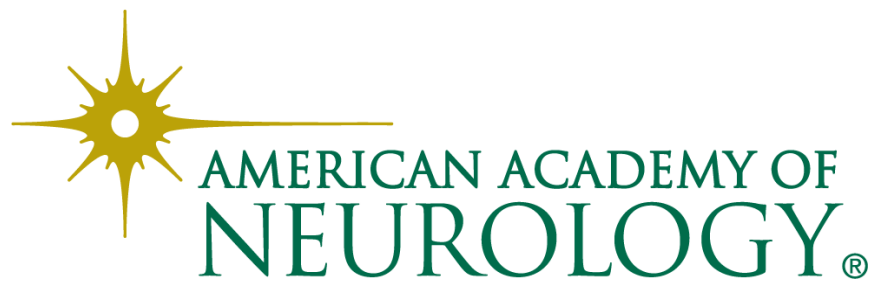

